\title{
FORAMINÍFEROS BENTÓNICOS AGLUTINADOS DE LOS DEPÓSITOS TURBIDÍTICOS. ÁREA NÁPOLES, SUR DE SAN MARCOS DE TARRAZÚ, COSTA RICA
}

\author{
AGGLUTINATED FORAMINIFERA FROM TURBIDITIC DEPOSITS, NÁPOLES \\ AREA, SOUTH OF SAN MARCOS, TARRAZÚ, COSTA RICA
}

\author{
Lolita Campos*, Julliet Betancur \& Luis G. Obando \\ Escuela Centroamericana de Geología, Universidad de Costa Rica \\ Apdo. 214-2060 UCR, Costa Rica \\ *Autora para contacto: lolita.campos@ucr.ac.cr
}

(Recibido: 11/07/2012 ; aceptado: 17/12/2012)

\begin{abstract}
In the area of Napoles, South of San Marcos, Tarrazú, located within a broad structural belt not yet fully defined at the boundary between and Térraba and Valle Central sedimentary basins, the sample LOR-10 provided an faunal assemblage of exclusively agglutinated benthic foraminifera. As there were not found planktonic foraminifera, biostratigraphic determinations were not possible to obtain. Of the identified individuals, these correspond to 3 suborders, 9 superfamilies and 33 species. Regarding to the Shannon diversity index $(H)$, the result for paleoecological interpretations was of $\mathrm{H}=1.4$, indicating conditions of marshes and marginal marine environments. On the other hand, the benthic foraminifera identified in the sample to species level, have very wide ranges of existence: from Triassic to Recent.

From the point of view regarding to paleoecological salinity, there were determined the following percentages: rotaliids $53.3 \%$, texturaliids $41.9 \%$ and miliolids $2.2 \%$, values that are indicative of brackish lagoon environments, estuarine and shelf, these mix of environments is indicative of an allochthonous reworked deposit.

The presence of Portatrochammina sp. (4.3\%) that appears between 500 and $2000 \mathrm{~m}$, but is abundant approximately between 600 and $700 \mathrm{~m}$ and of Cibicides lobatulus (3.2\%) indicative of the upper middle bathyal zone (500-1500 $\mathrm{m})$, confirm the interpretation of the deposit environment as a submarine fan of middle bathyal waters. Likewise, the preeminence of agglutinated foraminifera suggests an important contribution of detritus into the basin. Finally, stratified, cold, deep, basins with high sedimentation rates favor the preservation of agglutinated foraminifera instead carbonate ones.

Keywords: San Marcos, Terraba sedimentary basin boundary, Valle Central sedimentary basin boundary, Paleocene, agglutinated foraminifera, turbidites, Morozovella velascoensis.
\end{abstract}




\begin{abstract}
RESUMEN: En el sector de Nápoles, San Marcos de Tarrazú, situado dentro de una ancha faja estructural aún no completamente definida en el límite entre las cuencas sedimentarias de Valle Central y Térraba, la muestra LOR-10 brindó una asociación faunística de exclusivamente foraminíferos bentónicos. Como no fueron encontrados foraminíferos planctónicos no se pudieron realizar determinaciones bioestratigráficas. Del total de individuos identificados, estos corresponden a 3 subórdenes, 9 superfamilias y 33 especies. Con respecto al índice de diversidad de Shannon $(\mathrm{H})$, para interpretaciones paleoecológicas el resultado fue de $\mathrm{H}=1.4$, indicando condiciones de ciénagas y ambientes marginales marinos. Los foraminíferos bentónicos identificados hasta nivel de especie en la muestra, poseen rangos de existencia muy amplio señalando una zona que se extiende del Triásico al Reciente.

Desde el punto de vista paleoecológico en relación con la salinidad, fueron determinados los siguientes porcentajes $53.3 \%$ de rotaliinos, $41.9 \%$ de texturaliinos y $2.2 \%$ de miliólidos, valores que son indicadores de ambientes de laguna salobre, estuario y plataforma, esta mezcla de fauna de ambientes distintos sugiere que se trata de un depósito alóctono retrabajado.

La identificación de Portatrochammina sp. (4.3\%) que aparece entre los 500 y $2000 \mathrm{~m}$, pero es abundante aproximadamente entre los 600 y $700 \mathrm{~m}$ y de Cibicides lobatulus (3.2\%) indicadora de la zona batial media superior (500-1500 m), confirman la interpretación del ambiente del depósito como un abanico submarino de talud del ámbito batial medio. Así mismo la preeminencia de los foraminíferos aglutinados sugiere un importante aporte de detrito hacia la cuenca. Por último, las cuencas estratificadas, frías, profundas, con altas tasas de sedimentación favorecen la preservación de las asociaciones de foraminíferos aglutinados sobre los carbonatados.
\end{abstract}

Palabras clave: San Marcos, Cuenca sedimentaria Térraba, cuenca sedimentaria Valle Central, Paleoceno, foraminíferos aglutinados, turbiditas, Morozovella velascoensis.

\section{INTRODUCCIÓN}

En el contexto del proyecto de investigación "Transectos para la delimitación del límite noroeste de la cuenca Térraba" No. 113-A9-060, de la Vicerrectoría de Investigación de la Universidad de Costa Rica, se han realizado una serie de transectos geológicos con énfasis en el levantamiento estratigráfico en el área limítrofe entre las cuencas sedimentarias Valle Central y Térraba (Astorga et al, 1991; Campos 2001), con el fin de situar de manera más precisa el borde entre ambas y establecer el modelo estratigráfico de la zona, la cual se encuentra afectada por una tectónica compleja manifiesta por la presencia de grandes fallas inversas y de desplazamiento de rumbo. De la totalidad de las muestras tomadas en el transecto: San Lorenzo-NápolesChilamate, esto al sur de San Marcos de Tarrazú, sólo la muestra LOR-10 no resultó estéril. La ubicación geográfica y estratigráfica de la misma se muestra en la figura No.1. Dado que estructuralmente la serie rocosa donde se sitúa la muestra LOR-10 sobreyace tectónicamente la muestra A04 datada con
Morozovella velascoensis como Paleoceno Tardío (Obando, 2011), se desconoce si la muestra LOR-10 es de edad Paleoceno Superior o Inferior.

\section{MATERIALES Y MÉTODOS}

Se utilizó este método de extracción para los microfósiles: las muestras se quebraron y se siguió el procedimiento estándar de agua oxigenada $\left(\mathrm{H}_{2} \mathrm{O}_{2}\right)$ al $10 \%$, al cabo de aproximadamente 24 horas se tamizaron (tamices 30, 60, $200 \mu \mathrm{m}$ ) y las diferentes fracciones fueron secadas en recipientes separados y luego analizadas al microscopio. Adicionalmente, se procedió a realizar el procedimiento descrito por Lirer (2000), el cual consiste en la dilución de ácido acético $\left(\mathrm{CH}_{3} \mathrm{COOH}\right)$ al $80 \%$, durante un tiempo de disgregación de 2 horas de la muestra en este ácido. Posteriormente se observó la fracción media al microscopio para determinar si estaban listas para el análisis faunístico, de lo anterior se concluye que para ninguna de las muestras se observaron características reconocibles en los 

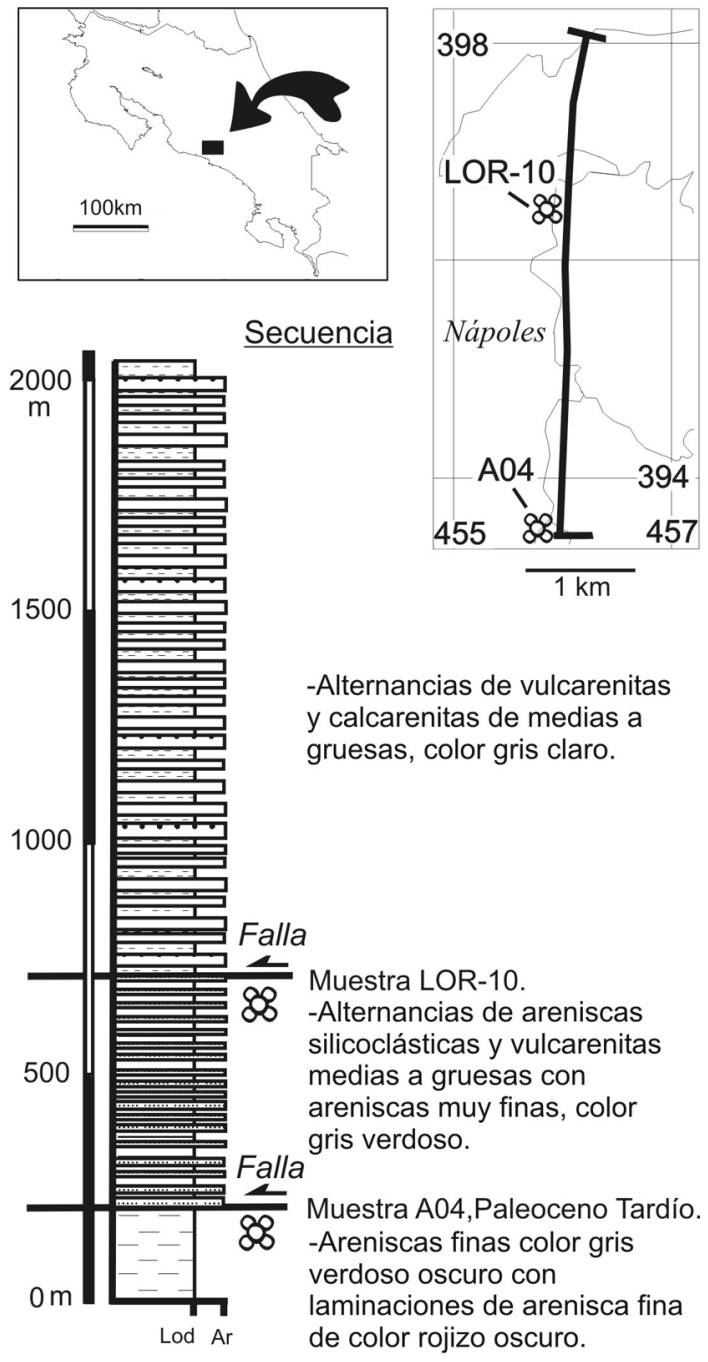

Fig.1: Columna estratigráfica, transecto San LorenzoNápoles-Chilamate, se indica la ubicación estratigráfica de las muestras A04 del Paleoceno y la muestra LOR-10. Referencia hoja topográfica Dota, 1:50 000, IGN.

microfósiles, así que se procede al lavado sónico de la fracción media, cada 2 horas durante 1 día, cada lavado estuvo precedido del lavado en la malla $200 \mu \mathrm{m}$.

A la muestra LOR-10 (Fig.2) se le realizó una limpieza manual, como resultado se obtuvieron 375 especímenes, para cuya mayoría se identificó hasta nivel de género siguiendo a Loeblich \& Tappan $(1964,1988)$.Para los individuos que fueron identificados hasta nivel de especie, se siguió la bibliografía referenciada en el cuadro 1 . Se tomaron fotografías en el estereomicroscopio Leica EZ4D. Adicionalmente, se calculó el índice de diversidad de Shannon $(\mathrm{H})$, para interpretaciones paleoecológicas cuyo resultado fue de $\mathrm{H}=1.4$.

\section{RESULTADOS Y DISCUSIÓN}

Como ya se mencionó, la única muestra que brindó microforaminíferos en lavado fue la LOR-10. En esta muestra, la fauna presente está caracterizada exclusivamente por foraminíferos bentónicos. No se observan peloides fecales, ni meiofauna. Como no fueron encontrados foraminíferos planctónicos no se pudieron realizar determinaciones bioestratigráficas. Sin embargo, los foraminíferos bentónicos, además de ser excelentes marcadores paleobatimétricos, son útiles como marcadores del aporte de nutrientes y de las condiciones de oxigenación en el fondo marino, constituyendo por tanto una importante herramienta para inferir los cambios paleoambientales.

En la muestra LOR-10, las formas bentónicas identificadas y su abundancia se muestran en la Cuadro 1. Del total de individuos identificados, estos corresponden a 3 subórdenes, 9 superfamilias y 33 especies. Con respecto al índice de diversidad de Shannon $(\mathrm{H})$, para interpretaciones paleoecológicas el resultado fue de $\mathrm{H}=1.4$, indicando condiciones de ciénagas y ambientes marginales marinos (Alve et al., 2011).

Los foraminíferos bentónicos identificados hasta nivel de especie en la muestra, poseen según Loeblich \& Tappan (1964) un rango de existencia del Triásico al Reciente (Cuadro 2).

Desde el punto de vista paleoecológico en relación con la salinidad, fueron determinados los siguientes porcentajes $53.3 \%$ de rotaliinos, $41.9 \%$ de texturaliinos y $2.2 \%$ de miliólidos, valores que 
Cuadro 1

Identificación de especies, abundancias y referencias bibliográficas

\begin{tabular}{|c|c|c|c|c|c|}
\hline Sub orden & Super familia & Especies & Número de individuos & $\%$ & Ref. \\
\hline \multirow[t]{15}{*}{ ROT } & Rotaliacea & (1) Elphidium sp. & 17 & 4,5 & (1) \\
\hline & & (2) Pararotalia sp. & 6 & 1,6 & (1) \\
\hline & & (3) Cribroelphidium excavatum & 18 & 4,8 & (2) \\
\hline & & (4) Calcarina Calcar & 25 & 6,7 & (3) \\
\hline & Buliminacea & (5) Angulogerina muralis & 1 & 0,3 & (4) \\
\hline & Cassidulinacea & (6) Cassidulina reflexa & 42 & 11,2 & $(5)$ \\
\hline & Robertinacea & (7) Epistomina caracolla & 3 & 0,8 & (6) \\
\hline & Discorbacea & (8) Rosalina sp. & 19 & 5,1 & (1) \\
\hline & & (9) Nuttallinella sp. & 5 & 1,3 & (1) \\
\hline & Nodosariacea & (10) Lenticulina punctata & 2 & 0,5 & (1) \\
\hline & Orbitoidacea & (11) Cibicides lobatulus & 12 & 3,2 & (2) \\
\hline & & (12) Cibicides laurisae & 22 & 5,9 & (7) \\
\hline & & (13) Cibicides sp. & 23 & 6,1 & $(1)$ \\
\hline & & (14) Carpenteria monticularis & 5 & 1,3 & (8) \\
\hline & & Subtotal & 200 & 53,3 & \\
\hline \multirow[t]{3}{*}{ MIL } & Miliolacea & (15) Pyrgo sp. & 1 & 0,3 & (1) \\
\hline & & (16) Parrina sp. & 7 & 1,9 & (1) \\
\hline & & Subtotal & 8 & 2,2 & \\
\hline \multirow[t]{20}{*}{ TEXT } & Lituolacea & (17) Trochammina squamata & 15 & 4 & (2) \\
\hline & & (18) Trochammina sp. & 31 & 8,3 & $(1)$ \\
\hline & & (19) Trochammina sp1. & 7 & 1,9 & (9) \\
\hline & & (20) Trochammina yubarensis & 15 & 4 & (10) \\
\hline & & (21) Portatrochammina sp. & 16 & 4,3 & (11) \\
\hline & & (22) Clavulina carinata & 6 & 1,6 & (12) \\
\hline & & (23) Ammobaculites sp. & 17 & 4,5 & $(1$ \\
\hline & & (24) Ammobaculites sp1 & 15 & 4 & (1) \\
\hline & & (25) Ammobaculites sp2 & 1 & 0,3 & (1) \\
\hline & & (26) Haplophragmoides sp. & 9 & 2,4 & (1) \\
\hline & & (27) Haplophragmoides sp1. & 5 & 1,3 & (1) \\
\hline & & (28) Reticulophragmium rotundidorsarum & 1 & 0,3 & (12) \\
\hline & & (29) Ammomarginulina troptunensis & 15 & 4 & (13) \\
\hline & & (30) Reophax cf. R. excentricus & 4 & 1,1 & (13) \\
\hline & & Subtotal & 157 & 41,9 & \\
\hline & & (31) Especie sin diferenciar 1 & 1 & 0,3 & \\
\hline & & (32) Especie sin diferenciar 2 & 5 & 1,3 & \\
\hline & & (33) Especie sin diferenciar 3 & 4 & 1,1 & \\
\hline & & Subtotal & 10 & 2,6 & \\
\hline & & ABUNDANCIA & 375 & 100 & \\
\hline
\end{tabular}


Referencias (Ref): (1)Loeblich \& Tappan, 1964-1988, (2)Thomas et al., 1990, (3)Devi Gadi \& Rajashekhar, 2009, (4)Ortiz \& Thomas, 2006, (5)Katz \& Miller, 1983, (6)Williamson \& Stam, 1988, (7)Nomura, 1988, (8)Akimoto, 1990, (9)Miller et al., 1982, (10)Scout et al.,2000, Palaeo-electronica.org, (11)Fillon, 1974, (12)Govindan, 2004, (13)Hanagata, 2003.

según Brasier (1980) son indicadores de ambientes de laguna salobre, estuario y plataforma, esta mezcla de fauna de ambientes distintos sugiere que se trata de un depósito alóctono retrabajado, conclusión acorde con la interpretación sedimentológica de la sucesión sedimentaria de donde procede la muestra como depósito de abanico submarino.

La mezcla de ambientes está indicada además por los siguientes criterios; el alto porcentaje de especímenes bentónicos aglutinados, cuya presencia se favorece en ambientes con escasa concentración de $\mathrm{CaCO}_{3}$, características oligotróficas, y fuertemente afectados por influjos de agua fresca, sugiriendo así ambientes de laguna y estuario. Además están presentes las siguientes especies: a) Ammobaculites sp. (8,8\%), Reophax sp. $(1,1 \%)$. Esta última ha sido encontrada tanto en ambientes de pantano y estuario, como en zonas de plataforma (Hanagata, 2003), b) Elphidium sp. $(4,5 \%)$, señala la zona litoral y nerítica $(0$ $48 \mathrm{~m}$ ) con ambientes de turbulencia de olas y una amplia fluctuación en la temperatura, c) los géneros Cassidulina $(11,2 \%)$ y Cibicides $(15,2 \%)$ que se asocian con guijarros, arena y limo con abundantes fragmentos de conchas, se presentan en bancos submarinos o sobre viejos sedimentos truncados, o como fauna relicta del movimiento del agua sobre elevaciones son indicadores de la zona nerítica externa (99-190m). Los planctónicos deberían acompañar esta asociación (Bergen \& O’Neil, 1979). d) Las zonas batiales superiores (200-500m) han sido indicadas por la presencia de las formas: Haplophragmoides $(3,7 \%)$, Reticulophragmium (0,3\%), Reophax (1,1\%) (Filipescu \& Beldean, 2008), Ammobaculites (8,8\%) (Govindan, 2004), Trochamminasquamata (4\%) (Ingle, et al., 1980).

Usualmente, los planctónicos acompañan la asociación de foraminíferos bentónicos aglutinados de mar profundo, aunque tienen una relación inversa con ellos (Filipescu \& Beldean, 2008). Cuando se tiene una proporción de aglutinados mayor o igual al $75 \%$, el porcentaje de planctónicos es casi cero y viceversa. Esta distribución de los planctónicos puede ser debida al transporte por las corrientes del fondo y porque de los bentónicos sólo permanecieron los aglutinados. La proporción de aglutinados respecto de los calcáreos incrementa con la profundidad, indicando una mayor

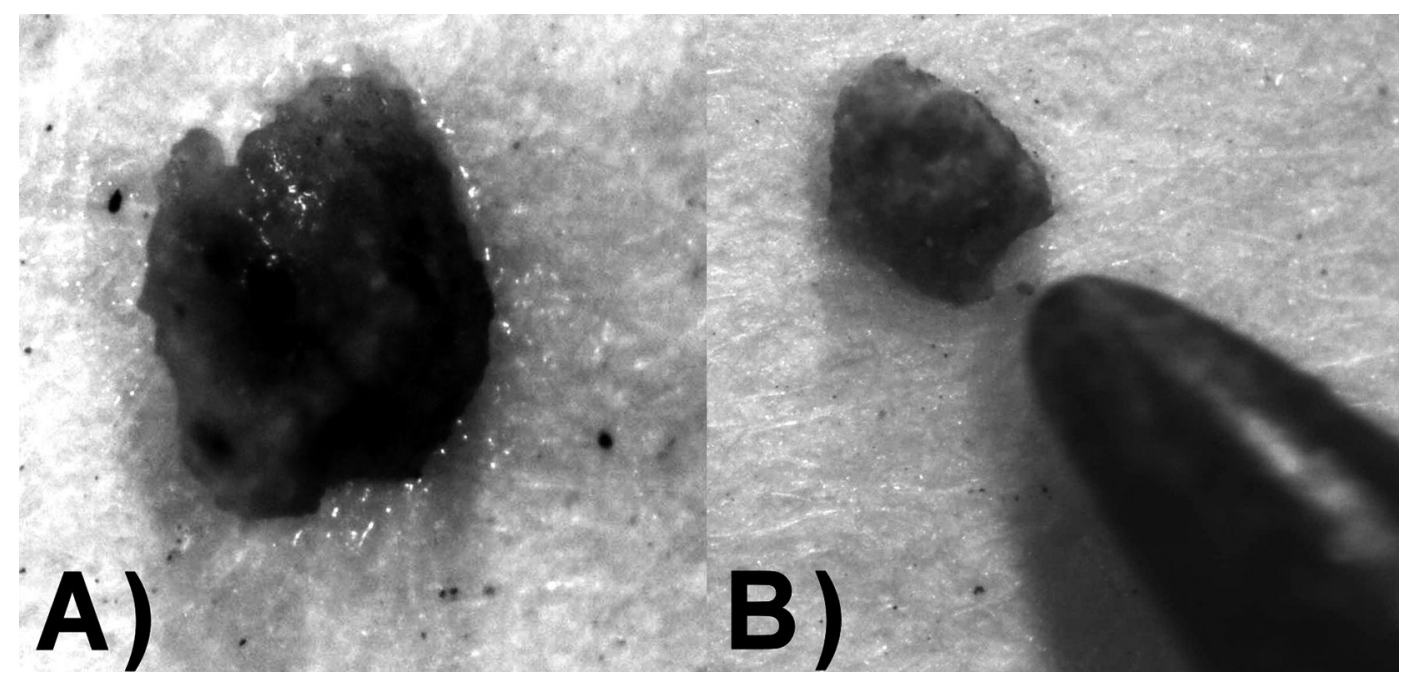

Fig. 2: Microfósiles de la fracción media de la muestra LOR10, A) Estado original del microfósil. B) Separación con un punzón. 
Rangos de edad de las especies (Véase el Cuadro 1 para identificar la especie referenciada)

\begin{tabular}{|c|c|c|c|c|c|c|c|c|c|}
\hline \multirow[t]{11}{*}{ Reciente } & Plioceno & Mioceno & Oligoceno & Eoceno & Paleoceno & Cretácico & Jurásico & Triásico & Especie \\
\hline & & & & & & & & & 3 \\
\hline & & & & & & & & & 4 \\
\hline & & & & & & & & & 5 y 6 \\
\hline & & & & & & & & & 7 \\
\hline & & & & & & & & & 10 \\
\hline & & & & & & & & & 11 \\
\hline & & & & & & & & & 14 \\
\hline & & & & & & & & & 17 \\
\hline & & & & & & & & & 22 \\
\hline & & & & & & & & & 29 \\
\hline
\end{tabular}

adaptabilidad de los arenáceos (Bergen \& O’Neil, 1979). e) La identificación de Portatrochammina sp. (4.3\%) que aparece entre los 500 y $2000 \mathrm{~m}$, pero es abundante aproximadamente entre los 600 y $700 \mathrm{~m}$ (Fillon, 1974) y de Cibicides lobatulus (3.2\%) (Ingle et al., 1980) indicadora de la zona batial media superior (500-1500m), confirman la interpretación del ambiente del depósito como un abanico submarino de talud del ámbito batial medio. Así mismo la preeminencia de los foraminíferos aglutinados sugiere un importante aporte de detrito hacia la cuenca. Esta condición del agua de la cuenca con abundante detrito, que depositó series sedimentarias terrígenas de altos espesores del orden de los cientos de metros, ha debido diluir la concentración de carbonato de calcio en el medio favoreciendo a las formas con conchilla aglutinada.

Dado que estructuralmente la serie rocosa donde se sitúa la muestra LOR-10 sobreyace tectónicamente la muestra A04 datada con Morozovella velascoensis como Paleoceno Tardío (Obando, 2011), se desconoce si la muestra LOR10 es de edad Paleoceno Superior o Inferior. No obstante, la carencia observada de fauna de foraminíferos planctónicos podría estar relacionada con los efectos de la extinción masiva del límite Cretácico/Paleógeno $(\mathrm{K} / \mathrm{Pg})$, cuyos drásticos cambios biológicos, oceánicos y climáticos afectaron extensamente a los foraminíferos planctónicos y el nannoplancton calcáreo que sufrieron importantes extinciones en masa en el limite K/ Pg y estuvieron sujetos a baja diversidad y abundancia a comienzos del Paleoceno, mientras que los foraminíferos bentónicos muestran cambios temporales en la composición de sus asociaciones, extinción de sólo unas pocas especies, y permanecieron comunes tras el límite K/Pg (Alegret Badiola, 2008).

Otras posibles causas para explicar la presencia de foraminíferos bentónicos aglutinados mencionadas en la literatura, son el de ser favorecidos por ambientes hipóxicos y con altas tasas de sedimentación (Nagy \& Seidenkrantz, 2003), con relación a bajas salinidades (Govindan, 2004). La asociación de los aglutinados con altas tasas de sedimentación y la ausencia de planctónicos y radiolarios se conoce en otras cuencas sedimentarias, p.e. en la Krishna-Godovari en la India, donde se asocian con arenas turbidíticas de $500 \mathrm{~m}$ de profundidad. El aumento de planctónicos se ha relacionado con el aumento en la profundidad de agua $(>1500 \mathrm{~m})$ (Govindan, in ibid).

Otro aspecto a considerar es el relacionado con el grado de oxigenación de las aguas, así en el Mar de Japón, durante el Mioceno, condiciones de aguas del fondo frías, deficientes en oxígeno, oligotróficas, con baja saturación en carbonatos y con comunicación limitada con el Océano Pacífico favorecieron el predominio de los foraminíferos aglutinados. Contrastantemente, dichas 
aguas del fondo durante el Plioceno fueron óxicas, ocasionalmente cálidas y saturadas de $\mathrm{CaCO}_{3}$, resultado de la comunicación entre los dos cuerpos de agua, el Mar de Japón y el océano Pacífico, posiblemente por un aumento del nivel del mar que favoreció la presencia de foraminíferos planctónicos (Hanagata, 2003). En resumen, las cuencas estratificadas, frías, profundas, con altas tasas de sedimentación favorecen la preservación de las asociaciones de foraminíferos aglutinados.

\section{AGRADECIMIENTOS:}

Se agradece a la Vicerrectoría de Investigación, Universidad de Costa Rica por los fondos aportados al proyecto de investigación, "Transectos para la delimitación del límite noroeste de la cuenca Térraba", No. 113-A9-060 y extensivo a todas aquellas personas que de alguna u otra manera han contribuido en la realización del presente trabajo.

\section{REFERENCIAS BIBLIOGRÁFICAS}

ALEGRET, L. 2008. Evolución paleoambiental durante el tránsito Cretácico/ Paleógeno basada en foraminíferos bentónicos.- Rev. Española Paleont. 23(1): 15-30.

ALVE, E., MURRAY, J.W. \& SKEI, J., 2011: Deep-sea benthic foraminifera, carbonate dissolution and species diversity in Hardangerfjord, Norway: An initial assessment.- Estuarine, Coastal and Shelf.Science, 92: 90-102.

AKIMOTO, K., 1990: Distribution of recent benthic foraminiferal faunas in the Pacific off southwest Japan and around Hachijojima Island.- Science Report Tohoku University Sendai, 60(2): 139-223.

ASTORGA, A., FERNÁNDEZ, J.A., BARBOZA, G., CAMPOS, L., OBANDO, J., AGUILAR, A., OBANDO, L.G., 1991:
Cuencas sedimentarias de Costa Rica: Evolución geodinámica y potencial de hidrocarburos.- Rev. Geol. Amér. Central, 13: 71-82.

BERGEN, F.W., \& O’NEIL, P., 1979: Distribution of Holocene foraminifera in the Gulf of Alaska.- J. Paleont. 53(6): 1267-1292.

BRASIER, M.D., 1980: Microfossils.- 193 págs. George Allen \& UNWIN, Londres.

CAMPOS, L., 2001: Geology and basins history of middle Costa Rica: an intraoceanic island arc in the convergence between the Caribbean and the Central Pacific plates.- 138 págs. University of Tuebingen, Alemania [Tesis Ph. D.]

DEVI GADI, S., \& RAJASHEKHAR, K.P., 2009: Monsoon-related periodicity in diversity and abundance of estuarine and non-estuarine foraminifera in the west coast of India.- $7^{\text {th }}$ Asia Pacific Marine Biotechnology Conference, Nat. Inst. Oceanography. Goa, India: 101.

FILIPESCU, S. \& BELDEAN, C., 2008: Foraminifera in the deep-sea environments of the Lower Hida Formation (Transylvanian Basin, Romania).- Acta Paleont. Romaniae, 6:105-114.

FILLON, R., 1974: Late Cenozoic foraminiferal paleoecology of the Ross Sea, Antarctica.Micropaleont. 20(2): 129-151.

GOVINDAN, A., 2004: Miocene deep-water agglutinated foraminifera from offshore Krishna-Godovari Basin, India.Micropaleont. 50(3): 213-252.

HANAGATA， S., 2003: Miocene-Pliocene Foraminifera from the Niigata Oil Fields Region, Northeastern Japan.Micropaleont. 49(4): 293-340. 
INGLE, J., KELLER, G., \& KOLPACK, R., 1980: Benthic foraminiferal biofacies, sediments and water masses of the southern Peru-Chile Trench area, sotheastern Pacific Ocean.-Micropaleont. 26(2):113-150.

KATZ, M., \& MILLER, K., 1983: Neogene benthic foraminiferal biofacies of the New Jersey transect.- Deep Sea Drilling Project [Initial Reports], XCV: 299-311.

LIRER, F., 2000: A new techique for retrieving calcareous microfossils from lithified lime deposits.- Micropaleont. 46(4): 365-369.

LOEBLICH, A.R. \& TAPPAN, H., 1964: Sarcodina and Foraminifera.- En: MOORE, R.C. (ed.): Treatise on invertebrate paleontology.- Kansas Univ. \& Geol. Soc. Amer. Lawrence: 1-900.

LOEBLICH, A.R. \& TAPPAN, H., 1987: Foraminiferal genera and their classification.- 869 págs. Van Nostrand Reinhold Co. New York.

MILLER, K., GRADSTEIN, F., \& BERGGREN, W., 1982: Late Cretaceous to early Tertiary agglutinated benthic foraminifera in the Labrador Sea.- Micropaleont. 28(1): 1-30.

NAGY, J. \& SEIDENKRANTZ, M. 2003: New foraminiferal taxa and revised biostratigraphy of Jurassic marginal marine deposits on Anholt, Denmark.- Micropaleont. 49(1): 27-46.

NOMURA, R., 1988: Paleoceanography of upper Maestrichtian to Eocene benthic foraminiferal assemblages at sites 752, 753 y 754, Easter Indian Ocean.- Proceeding of the Ocean Drilling Program, Sci. Results, 121: 3-75.
OBANDO, L. 2011: Estratigrafía y tectónica de la parte noreste de la hoja Dota (1:50 000), Costa Rica.- Rev. Geol. Amér. Central, 44: 71-82.

ORTIZ, S., \& THOMAS, E., 2006: LowerMiddle Eocene benthic foraminifera from The Fortuna Section (Betic Cordillera, southeastern Spain).- Micropaleont. 52(2): 97-150.

SCOUT. D., TAKAYANAGI, Y., HASEGAWA, S. \& SAITO, T., 2000: Illustration and taxonomic reevaluation of Neogene foraminifera described from Japan. Taxonomic Listing.- http://palaeoelectronica.org. http://palaeo-electronica.org/2000_2/foram/13_259.htm. [Consulta: 20 de junio de 2012].

THOMAS, F., MEDIOLI, F., \& SCOTT, D., 1990: Holocene and Latest Wisconsinan benthic foraminiferal assemblages and paleocirculation history, lower Scotian slope and rise.- J. Foraminiferal Res. 20(3): 212-245.

THOMAS, E., GAPOTCHENKO, T., VAREKAMP, J., MECRAY, E., \& BUCHHOLTZ TEN BRINK, M. 2000. Maps of benthic foraminiferal distribution and environmental changes in Long Island Sound between the 1940s and the 1990s.USGS CHAPTER 9. http://pubs.usgs.gov/ of/2000/of00-304/htmldocs/chap09/index. htm. [Consulta: 20 de junio de 2012].

WILLIAMSON, M. A., STAM, B., 1988: Jurassic/Cretaceous Epistominidae from Canada and Europe.- Micropaleont. 34(2): 136-158. 